\title{
Increased sensitivity in proton transfer reaction mass spectrometry by incorporation of a radiofrequency ion funnel
}

\author{
Shane Barber ${ }^{1}$, Robert S. Blake, ${ }^{1}$ Iain R. White, ${ }^{1}$ Paul S. Monks ${ }^{1}$, \\ Fraser Reich ${ }^{2}$, Steve Mullock ${ }^{2}$, Andrew M. Ellis ${ }^{1, *}$ \\ 1 Department of Chemistry, University of Leicester, University Road, Leicester LE1 7RH, UK \\ 2 Kore Technology Ltd, Cambridgeshire Business Park, Ely, Cambridgeshire CB7 4EA, U.K.
}

* Email: Andrew.Ellis@le.ac.uk

Manuscript submitted to Analytical Chemistry 


\begin{abstract}
A drift tube capable of simultaneously functioning as an ion funnel is demonstrated in proton transfer reaction mass spectrometry (PTR-MS) for the first time. The ion funnel enables a much higher proportion of ions to exit the drift tube and enter the mass spectrometer than would otherwise be the case. An increase in the detection sensitivity for volatile organic compounds of between one and two orders of magnitude is delivered, as demonstrated using several compounds. Other aspects of analytical performance explored in this study include the effective $E / N$ and dynamic range over which the drift tube is operated. The dual purpose drift tube/ion funnel can be coupled to various types of mass spectrometer to increase the detection sensitivity and may therefore offer considerable benefits in PTR-MS work.
\end{abstract}




\section{INTRODUCTION}

Introduced in the mid 1990s, proton transfer reaction mass spectrometry (PTR-MS) has become an increasingly important technique for the detection and monitoring of volatile organic compounds (VOCs) in air. ${ }^{1-5}$ It possesses a high sensitivity, allowing routine detection of VOCs at low parts per billion by volume (ppbV) and extending well into the parts per trillion by volume (pptV) regime for the best available instruments. This ability to distinguish trace organic gases from the vastly larger quantities of inorganic gases in air, such as $\mathrm{N}_{2}$ and $\mathrm{O}_{2}$, derives from the use of proton transfer, most commonly from $\mathrm{H}_{3} \mathrm{O}^{+}$, as the source of ions. This ionization method is selective to those gas constituents with a proton affinity exceeding that of $\mathrm{H}_{2} \mathrm{O}$, which rules out any contribution from the common inorganic constituents of air, which would otherwise generate large background ion signals that would severely diminish the detection sensitivity for VOCs. As well as being sensitive, PTR-MS is also quantitative. The latter property derives from the use of a drift tube reactor, which brings the $\mathrm{H}_{3} \mathrm{O}^{+}$into contact with the analyte for a fixed reaction time.

The PTR-MS technique was originally developed by the group of Werner Lindinger and coworkers. ${ }^{6}$ The earliest instruments coupled the ion source and drift tube with a quadrupole mass spectrometer (QMS). However, while compact and straightforward to use, QMS has several weaknesses when compared to other mass selective techniques. These include a relatively low mass resolution and a low operational efficiency, the latter arising from the fact that all ions, except those of a specific $\mathrm{m} / \mathrm{z}$, are rejected by the quadrupole mass filter at any instant in time. These deficiencies have seen other mass spectrometric techniques being interfaced with PTR-MS in recent years, including quadrupole ion traps ${ }^{7-10}$, linear ion traps ${ }^{11}$ and time-of-flight mass spectrometry. ${ }^{12-15}$ Timeof-flight mass spectrometry (TOF-MS) is particularly promising because it is a multichannel device capable of detecting all ions simultaneously. Moreover, with suitably folded ion trajectories, as seen in the standard reflectron and other more complex folded designs, ${ }^{15}$ a sufficiently high mass resolution can be achieved to distinguish many nominally isobaric compounds.

The performance of the mass spectrometer is critical to the ultimate detection sensitivity available in PTR-MS and is a topic that has been discussed in detail elsewhere. ${ }^{5}$ However, sensitivity is not determined solely by the mass analyzer and detector. Another factor which critically limits the 
detection sensitivity derives from the small exit aperture at the end of the drift tube: this aperture is necessary to maintain a sufficiently low pressure in the mass spectrometer while sustaining a pressure in the drift tube which is several orders of magnitude higher. Ordinarily, the vast majority of ions traversing the drift tube do not exit through this aperture, and so a large quantity of potentially useable ion signal is wasted.

In this paper we describe a means for dramatically increasing the proportion of ions that can pass from the drift tube into the mass spectrometer. The challenge is to take ions that are flowing through a region of relatively large volume and at a pressure of a few mbar (a typical pressure for a PTR-MS drift tube) and channel them through a small aperture into a much lower pressure zone. This challenge is not unique to PTR-MS: for example, it is encountered in mass spectrometers equipped with an electrospray ionization source. The electrospray source delivers liquid at atmospheric pressure into an electrically charged capillary and the subsequent formation of ions as the analyte leaves the capillary occurs in a region where the optimum pressure is a few mbar. A series of differential pumping stages can then be employed to eventually deliver the ions to a mass spectrometer. The loss of ions through these pumping stages can be mitigated to some extent by the use of one or more multipole ion guides, but major losses still occur. In particular, ion guides are excellent for the collisional focusing of already relatively narrow beams of ions but are ineffective for diffuse ion sources.

A novel solution to this problem was developed by Smith and co-workers in the late 1990s: the radiofrequency (RF) ion funnel. ${ }^{16}$ This RF funnel uses a series of electrodes with progressively reducing aperture sizes. A DC electrical field drives the ions axially through the funnel towards the exit aperture. In addition, an AC electric field is provided at a radiofrequency (RF) such that adjacent electrodes have opposite voltage polarities in the AC component. The RF field creates a strongly repulsive effective potential near the surface of each electrode which, coupled with the progressively diminishing aperture size, serves to focus the ions radially. This focusing effect can deliver a large increase in ion flux through the exit aperture and thus a large increase in signal at a downstream mass spectrometer. Various modifications have been made to the ion funnel design ${ }^{17-20}$ since its initial 
introduction and this has been supported by an improved understanding of the ion motion through trajectory simulations. ${ }^{17,19,21,22}$

Here the RF ion funnel concept is combined with PTR-MS for the first time. We were prompted to attempt this coupling because the optimum pressure for operation of an RF ion funnel is close to the typical operating pressure of a drift tube in PTR-MS. This suggests that it might be possible to construct a compact drift tube which can simultaneously operate as an ion funnel and as an ion-molecule reaction vessel with a fixed reaction time. This idea has been proposed and demonstrated previously in the context of ion mobility spectrometry ${ }^{23}$ but not for PTR-MS. In this paper we describe a prototype design and explore its initial application in PTR-MS. An improvement in the detection sensitivity of between one and two orders of magnitude is observed for most of the test gases, which suggests that the RF ion funnel/drift tube may significantly enhance the performance of PTR-MS.

\section{EXPERIMENTAL}

\section{The standard PTR-MS apparatus}

The PTR-MS instrument consists of a hollow cathode discharge ion source, a drift tube and ion transfer lens assembly, and a reflectron TOF-MS. $\mathrm{H}_{3} \mathrm{O}^{+}$ions are generated via a DC electrical discharge through water vapor. After passing through a source drift region, which maximizes the production of $\mathrm{H}_{3} \mathrm{O}^{+}$, the ions then pass through a $2 \mathrm{~mm}$ aperture into the drift tube, where they encounter the analyte. The drift tube is pumped by a mechanical pump with an effective speed of $\sim 31$

$\mathrm{s}^{-1}$ and a pressure of $\sim 1$ mbar is maintained in this region under normal operating conditions.

In the standard PTR-MS version of this instrument the drift tube consists of a series of equally spaced disk electrodes with a central aperture of $40 \mathrm{~mm}$ diameter. This arrangement delivers a uniform electric field along the drift tube. In the current study the electrode structure is modified to allow part of the drift tube to act as an ion funnel, as described in a separate section below. At the end of the drift tube is a $400 \mu \mathrm{m}$ orifice, which allows ions to enter the ion transfer zone for the TOF-MS. This ion transfer assembly consists of collection/collimating optics and feeds ions into the source 
region of a reflectron TOF-MS. The reflectron, which is pumped by a $701 \mathrm{~s}^{-1}$ turbomolecular pump, has a total effective ion flight path of $\sim 1.2 \mathrm{~m}$. Ions are detected at the end of their trajectory by a large entrance $(8 \mathrm{~mm} \times 32 \mathrm{~mm})$ discrete dynode detector. The ion signal is then amplified and fed into a proprietary pulse counting system for data acquisition, which consists of a time-to-digital converter interfaced to a PC. Data display and processing was carried out using the GRAMS software package.

\section{Ion funnel/drift tube}

The ion funnel consists of 29 stainless steel plates of $0.2 \mathrm{~mm}$ thickness, mounted on precision machined ceramic rods at an even spacing of $3.2 \mathrm{~mm}$ per plate. The orifice diameters of the plates through the first half of the stack is $40 \mathrm{~mm}$, as used in the standard drift tube reactor. In the second half of the drift tube the orifice diameter steadily decreases to $6 \mathrm{~mm}$ at the final plate before the exit orifice. To avoid the trapping of ions in axial potential wells, particularly those with low $\mathrm{m} / \mathrm{z}$, the following condition must be satisfied: ${ }^{24}$

$$
2 \pi \frac{\rho}{\delta} \exp \left(\frac{-2 \rho}{\delta}\right)<<1
$$

where $\rho=$ electrode orifice diameter and $\delta=d / \pi$ where $d=$ electrode spacing. The above condition is easily satisfied for the electrode geometries used in this work.

A resistive divider consisting of a ceramic substrate patterned with thin film resistors is used to deliver the static voltages to individual plates, with vacuum feedthroughs providing connections to the ends of the divider. The DC voltage across the reactor may be varied between 30 and $450 \mathrm{~V}$. When operating in RF mode, the lower end of this scale gave the best results in terms of detection sensitivity. Capacitor chains are used to feed a balanced RF input voltage to the plates in the downstream half of the drift tube.

The RF voltage is provided by a simple proprietary AC generator whose output transformer is arranged to be in resonance with the load capacitance. This resonance is used to achieve a roughly sinusoidal waveform at around $800 \mathrm{kHz}$, with adjustable amplitude up to about $200 \mathrm{~V}$ peak to peak. 


\section{Operating conditions}

The ion funnel delivered a large increase in the $\mathrm{H}_{3} \mathrm{O}^{+}$ion count rate detected at $m / z=19$. For the optimized ion funnel a reading of $6 \times 10^{6} \mathrm{~Hz}$ was observed whereas in the optimized DC-only mode using exactly the same electrode configuration an $\mathrm{H}_{3} \mathrm{O}^{+}$count rate of $5 \times 10^{5} \mathrm{~Hz}$ was observed. The far higher ion count rate in the RF mode is exactly what we are seeking, but it also has the disadvantage of contributing to rapid degradation of the ion detector if allowed to persist, as well as overloading the time-to-digital converter. The TOF-MS detector was therefore equipped with a purpose-built gating system to prevent ions with $m / z=19$ from generating a cascade of electrons and hence wear in the detector. Instead, the $\mathrm{H}_{3} \mathrm{O}^{+}$count rate was deduced by monitoring the $\mathrm{H}_{3}{ }^{18} \mathrm{O}^{+}$signal at $m / z=21$ and using the known ${ }^{16} \mathrm{O}:{ }^{18} \mathrm{O}$ natural abundance ratio $(499: 1)$ to determine the count rate of $\mathrm{H}_{3} \mathrm{O}^{+}$. The detector gating was achieved by applying a negative going voltage pulse at dynode number two of the detector, rejecting electrons generated by the ion impact and thus eliminating wear from all dynodes except the first, the conversion dynode.

Analytes were injected into the drift tube at a typical flow rate of $85 \mathrm{ml} \mathrm{min}^{-1}$. To assess the analytical performance, a variety of VOCs at known concentrations were delivered in both the DConly and RF funnel modes of operation. The source of gases was a calibrated gas mixture containing methanol, acetaldehyde, trans-2-butene, acetone, methacrolein, cyclohexanone, and $\beta$-pinene at known concentrations (BOC Special Gases; nominal mixing ratio $1 \mathrm{ppmV}$ for each compound, with an estimated uncertainty of $\pm 10 \%$ ) in a balance gas of nitrogen. For our experiments this mixture was subjected to a 10 -fold static dilution to generate a new mixture with roughly $100 \mathrm{ppbV}$ of each component, with the balance gas being nitrogen. Subsequent dilutions were then performed dynamically by feeding the cylinder gas into a Kintek $491 \mathrm{M}$ and diluting in nitrogen.

\section{RESULTS AND DISCUSSION}

\section{Effective $E / N$}


Application of an electric field along the drift tube draws ions through the reaction zone and delivers a series of energetic ion-molecule collisions which serve to minimize the formation of cluster ions, particularly hydrated hydronium ions, $\mathrm{H}_{3} \mathrm{O}^{+}\left(\mathrm{H}_{2} \mathrm{O}\right)_{n}$. Without this feature both the mass spectrometric and the kinetic analysis would be excessively complex. The electrodes in a drift tube are normally equally spaced along the length of the tube, thus creating an axially uniform electric field. The standard means of expressing the drift tube conditions is through the quantity $E / N$, where $E$ is the electric field and $N$ is the number density of the gas. $E / N$ is most commonly expressed in the unit known as the Townsend (Td), where $1 \mathrm{Td}=10^{-17} \mathrm{~V} \mathrm{~cm}^{2}$.

For a standard drift tube containing gas at a known pressure and with a uniform DC electric field, $E / N$ is a well-defined quantity. A typical operating $E / N$ in PTR-MS is in the region of $120 \mathrm{Td}$. However, the effective $E / N$ of a combined ion funnel/drift tube, with its contribution from both DC and $\mathrm{AC}$ electric fields, is no longer obvious. We intend to provide a quantitative model of the effective $E / N$ in a separate publication. In the current study we simply take an empirical view of the effective $E / N$ by seeking operating conditions for the ion funnel/drift tube that match the performance of the same drift tube when operated under the optimum DC-only conditions (see below).

The performance criterion we use for this comparison is the degree of hydration of $\mathrm{H}_{3} \mathrm{O}^{+}$. As mentioned above, under ideal conditions for PTR-MS the $\mathrm{H}_{3} \mathrm{O}^{+}$ion is by far the dominant source of protons. In Figure 1 we show the signals observed in mass channels corresponding to both $\mathrm{H}_{3} \mathrm{O}^{+}$and $\mathrm{H}_{3} \mathrm{O}^{+}\left(\mathrm{H}_{2} \mathrm{O}\right)$ as a function of $E / N$ for the drift tube with the RF switched off. In this DC-only mode we see that the hydrated hydronium cluster ions dominate at low $E / N$ but $\mathrm{H}_{3} \mathrm{O}^{+}$becomes the most abundant ion at $E / N \approx 100 \mathrm{Td}$. Ideal conditions for operating the drift tube in this mode of operation would be at $E / N \approx 125 \mathrm{Td}$, since under these conditions less than $5 \%$ of $\mathrm{H}_{3} \mathrm{O}^{+}$is in its hydrated form. As mentioned previously this is a fairly typical $E / N$ value for PTR-MS and it shows that the modified electrode structure employed here, with the converging aperture sizes, does not have a major impact on the ion-molecule chemistry. Of course one could operate at an even higher $E / N$, which would further reduce $\mathrm{H}_{3} \mathrm{O}^{+}\left(\mathrm{H}_{2} \mathrm{O}\right)_{n}$ cluster levels relative to $\mathrm{H}_{3} \mathrm{O}^{+}$through increasingly energetic ion-molecule collisions. However, this is generally undesirable if taken too far because it leads to excessive 
fragmentation of protonated analyte ions and can lead to a decrease in sensitivity as the reaction time for proton transfer decreases.

Switching to the ion funnel mode, we now seek operating conditions when the RF field is also applied which deliver roughly the same $\left[\mathrm{H}_{3} \mathrm{O}^{+}\right] /\left[\mathrm{H}_{3} \mathrm{O}^{+}\left(\mathrm{H}_{2} \mathrm{O}\right)\right]$ ratio as seen when operating in the DConly mode. Figure 2 shows how this ratio is affected by the RF conditions. The quantity being varied here was the peak-to-peak amplitude of the AC voltage, i.e. the DC contribution was fixed. A full exploration of the effect of varying both the DC and $\mathrm{AC}$ conditions will be described in a future publication. Here the DC voltage across the drift tube was fixed at $100 \mathrm{~V}$, giving a DC-only contribution to the $E / N$ of $\sim 60 \mathrm{Td}$. The $\left[\mathrm{H}_{3} \mathrm{O}^{+}\right] /\left[\mathrm{H}_{3} \mathrm{O}^{+}\left(\mathrm{H}_{2} \mathrm{O}\right)\right]$ ratio varies systematically with applied RF voltage in a way that is qualitatively comparable to the DC-only case, with a plateau being reached at an RF voltage of roughly $150 \mathrm{~V}$ peak-to-peak. This suggests that the ion funnel behaves equivalently to a DC-only drift tube under these particular conditions, but with an effective $E / N$ that now depends on both the RF and DC electric fields. The same $\left[\mathrm{H}_{3} \mathrm{O}^{+}\right] /\left[\mathrm{H}_{3} \mathrm{O}^{+}\left(\mathrm{H}_{2} \mathrm{O}\right)\right]$ ratio as obtained under optimum DC-only conditions was obtained in the RF mode when the RF voltage was $170 \mathrm{~V}$. This RF voltage was used in all of the subsequent studies detailed below.

For illustration of the effect on VOCs, Figure 3 shows how the effective $E / N$ in the RF mode of operation, inferred from the water cluster ion measurements described above, affects the signal from acetaldehyde. The principal ion product is the protonated parent molecule $(\mathrm{m} / \mathrm{z} 45)$, but there is also a small quantity of a fragment at $\mathrm{m} / \mathrm{z} 27$; the latter is formed by dehydration of the protonated parent molecule. At an effective $E / N$ equivalent to $125 \mathrm{Td}$, the relative abundance of the dehydration fragment is slightly higher (7\%) than the corresponding measurement made in the DC-only mode (4\%), implying that the effective $E / N$, as calculated by the extent of reagent ion clustering, is a reasonable (but not perfect) measure of the ion-molecule collision conditions when the ion funnel is switched on.

\section{Detection sensitivity and limit of detection}

The instrument was calibrated by delivering gas from a commercially prepared standard gas mixture, as detailed earlier. This contained seven principal components detectable by PTR-MS: methanol, 
acetaldehyde, trans-2-butene, acetone, methacrolein, cyclohexanone and $\beta$-pinene. We define the detection sensitivity for these compounds as the ion count rate for a given quantity of supplied analyte. We use units of $\mathrm{Hz} \mathrm{ppbV}^{-1}$ and obtain this quantity, sometimes also known as the calibration factor, from a plot of ion count rate versus the delivered quantity of each gas. The gas mixture was dynamically diluted to deliver known concentrations of each compound ranging from the very low $\mathrm{ppbV}$ through to several hundred ppbV. For each compound the protonated parent species was monitored to generate the data shown in Figure 4. Of the seven compounds in the gas mixture, six show excellent linear relationships between the number of ion counts and delivered concentration, with linear correlation coefficients $\left(r^{2}\right)$ close to unity. The exception is methanol, but even here $r^{2} \approx$ 0.90. It seems likely that there is some interference with the protonated methanol signal caused by contributions from the neighbouring $\mathrm{O}_{2}^{+}$peak at $\mathrm{m} / \mathrm{z}=32$, which is observed because of some diffusion of analyte gas into the ion source region. Although small relative to $\mathrm{H}_{3} \mathrm{O}^{+}$the $\mathrm{O}_{2}{ }^{+}$signal still dwarves that from protonated methanol.

The sensitivities extracted from these plots are summarized in Table 1. Values are shown for both $\mathrm{DC}$ and RF modes of operation, along with the RF/DC sensitivity ratio. For the majority of compounds the switch from DC to RF mode improves the sensitivity by between one and two orders of magnitude, although this is exceeded for acetone, methacrolein and cyclohexanone.

The limit of detection in an analytical context is commonly defined as three times the background noise, i.e.

$$
\mathrm{LoD}=\frac{3[\mathrm{M}]}{(S / N)}
$$

where $S$ is the mean background signal level at the chosen $m / z, N$ is the corresponding noise level, and $[\mathrm{M}]$ is the delivered concentration of compound M. Contributions to the background signal can be mass specific and will include contributions from traces of compounds present in the instrument (e.g. from components used in the instrument construction, degassing, etc.), as well as in the gas employed for background measurements. A mass-dependent background noise level was determined without 
any added analyte by making repeat measurements of $S$ for a fixed integration time and then associating the standard deviation with $N$.

Table 1 compares the LoDs for the DC and RF modes of operation obtained for $20 \mathrm{~s}$ of data accumulation. There is a substantial improvement in LoD for all compounds in moving from DC-only operation to the RF mode. In almost all cases the LoD is reduced from several hundred pptV in the DC mode to $\leq 200 \mathrm{pptV}$ in the RF mode, and in some cases considerably better. The most marked exception is methanol, for reasons already identified above (contamination from $\mathrm{O}_{2}{ }^{+}$). Also, trans-2butene does not fare too well, with an LoD near $350 \mathrm{pptV}$ in the RF mode for $20 \mathrm{~s}$ data accumulation. This relatively poor LoD may partly be due to the comparatively low polarizabilty and the lack of a permanent electric dipole moment for this molecule, which will reduce the proton transfer rate when compared to other many other molecules in Table 1. Furthermore, trans-2-butene has the lowest proton affinity of all of the molecules in Table 1 and therefore will be more susceptible to backreaction with water vapor.

\section{CONCLUSIONS}

The drift tube of a PTR-MS instrument has been modified to allow it to operate as both a conventional drift tube and as an ion funnel. We have coupled this combined drift tube/ion funnel with a mass spectrometer for the first time and the resulting PTR-MS instrument has been shown to deliver a much higher proportion of ions out of the drift tube and into the source region of the mass spectrometer. The improvement in detection sensitivity in going from the DC mode to the RF mode is more than two orders of magnitude for the majority of VOCs considered in this work. The dual purpose drift tube/ion funnel could easily be coupled to other types of mass spectrometer to increase the detection sensitivity and may therefore offer considerable benefits in PTR-MS work.

The limit of detection (LoD) is currently restricted by persistent chemical background signals. The source of these signals would seem to be from materials incorporated within the instrument, rather than any extraneous sources in the laboratory. It should be possible to substantially reduce this background contamination to achieve a LoD in the single figure pptV range for $20 \mathrm{~s}$ of data accumulation with the current instrument, i.e. an improvement approaching two orders of magnitude 
relative to the DC-only mode, which would be compatible with the improvement already seen in the detection sensitivity.

\section{REFERENCES}

(1) Lindinger, W.; Hansel, A.; Jordan, A. Int. J. Mass Spectrom. Ion Process. 1998, 173, 191241.

(2) Lindinger, W.; Hansel, A.; Jordan, A. Chem. Soc. Rev. 1998, 27, 347-375.

(3) Hewitt, C. N.; Hayward, S.; Tani, A. J. Environ. Monit. 2003, 5, 1-7.

(4) de Gouw, J. A.; Warneke, C. Mass Spectrom. Rev. 2007, 26, 223-257.

(5) Blake, R. S.; Monks, P. S.; Ellis, A. M. Chem. Rev. 2009, 109, 861-896.

(6) Hansel, A.; Jordan, A.; Holzinger, R.; Prazeller, P.; Vogel, W.; Lindinger, W. Int. J. Mass Spectrom. Ion Process. 1995, 149/150, 609-619.

(7) Prazeller, P.; Palmer, P. T.; Boscaini, E.; Jobson, T.; Alexander, M. Rapid Commun. Mass Spectrom. 2003, 17, 1593-1599.

(8) Warneke, C.; de Gouw, J. A.; Lovejoy, E. R.; Murphy, P. C.; Kuster, W. C.; Fall, R. J. Am. Soc. Mass Spectrom. 2005, 16, 1316-1324.

(9) Steeghs, M. M. L.; Sikkens, C.; Crespo, E.; Cristescu, S. M.; Harren, F. J. M. Int. J. Mass Spectrom. 2007, 262, 16-24.

(10) Steeghs, M. M. L.; Crespo, E.; Harren, F. J. M. Int. J. Mass Spectrom.2007, 263, 204-212.

(11) Mielke, L. H.; Erickson, D. E.; McLuckey, S. A.; Müller, M.; Wisthaler, A.; Hansel, A.; Shepson P. B. Anal. Chem. 2008, 80, 8171-8177.

(12) Blake, R. S.; Whyte, C.; Hughes, C. O.; Ellis, A. M.; Monks, P. S. Anal. Chem. 2004, 76, 3841-3845.

(13) Ennis, C. J.; Reynolds, J. C.; Keely, B. J.; Carpenter, L. J. Int. J.Mass Spectrom. 2005, 247, $72-80$.

(14) Tanimoto, H.; Aoki, N.; Inomata, S.; Hirokawa, J.; Sadanaga, Y. Int. J. Mass Spectrom. 2007, $263,1-11$. 
(15) Jordan, A.; Haidacher, S.; Hanel, G.; Hartungen, E.; Maerk, L.; Seehauser, H.; Schottkowsky, R.; Sulzer, P.; Maerk, T. D. Int. J. Mass Spectrom. 2009, 286, 122-128.

(16) Shaffer, S. A.; Tang, K.; Anderson, G. A.; Prior, D. C.; Udseth, H. R.; Smith, R. D. Rapid Commun. Mass Spectrom. 1997, 11, 1813-1817.

(17) Shaffer, A. A.; Tolmachev, A.; Prior, D. C.; Anderson, G. A.; Udseth; H. R., Smith, R. D. Anal. Chem. 1999, 71, 2957-2964.

(18) Kim, T.; Tolmachev, A. V.; Harkewicz, Prior, D. C.; Anderson, G.; Udseth, H. R.; Smith, R. D. Anal. Chem. 2000, 72, 2247-2255.

(19) Julian, R. R.; Mabbett, S. R.; Jarrold, M. F. J. Am. Soc. Mass Spectrom. 2005, 16, 1708-1712.

(20) Ibrahim, Y.; Tang, K.; Tolmachev, A. V.; Shvartsburg, A. A.; Smith, R. D. J. Am. Soc. Mass Spectrom. 2006, 17, 1299-1305.

(21) Lynn, E. C.; Chung, M. -C.; Han, C. -C. Rapid Commun. Mass Spectrom. 2000, 14, 21292134.

(22) Tolmachev, A. V.; Kim, T.; Udseth, H. R.; Smith, R. D.; Bailey, T. H.; Futrell, J. H. Int. J. Mass Spectrom. 2000, 203, 31-47.

(23) Shammel Baker, E.; Clowers, B. H.; Li, F.; Tang, K.; Tolmachev, A. V.; Prior, D. C.; Belov, M. E.; Smith, R. D. J. Am. Soc. Mass Spectrom. 2007, 18, 1176-1187.

(24) Kelly, R. T.; Tolmachev, A. V.; Page, J. S.; Tang, K.; Smith, R. D. Mass Spectrom. Rev. 2010, 29, 294-312. 
Table 1 Comparison of sensitivities and limits of detection (LoD) for several VOCs ${ }^{\text {a) }}$

\begin{tabular}{lcccccc}
\hline $\begin{array}{c}\text { Compound } \\
(\boldsymbol{m} / \boldsymbol{z} \text { for } \boldsymbol{M H}+\mathbf{+}\end{array}$ & \multicolumn{2}{c}{$\begin{array}{c}\text { Raw sensitivity/Hz } \\
\mathbf{p p b V}^{-1}\end{array}$} & \multicolumn{4}{c}{$\begin{array}{c}\text { LOD/ } \\
\mathbf{p p t V}\end{array}$} \\
& DC mode & $\mathbf{R F}$ mode & RF/DC & DC mode & RF mode & RF/DC \\
\hline Methanol (33) & 1.8 & 15 & 8 & 6125 & 1054 & 6 \\
Acetaldehyde (45) & 4.8 & 218 & 45 & 486 & 161 & 3 \\
Trans-2-butene (57) & 0.7 & 38 & 54 & 930 & 346 & 3 \\
Acetone (59) & 5.8 & 1162 & 200 & 445 & 30 & 15 \\
Methacrolein (71) & 2.4 & 387 & 161 & 351 & 26 & 13 \\
Cyclohexanone (99) & 3.1 & 686 & 221 & 271 & 15 & 18 \\
$\beta$-Pinene (137) & 1.1 & 164 & 149 & 425 & 123 & 3 \\
\hline
\end{tabular}

a) The DC data was collected at an $E / N$ of $120 \mathrm{Td}$ and the RF data was likewise obtained at effective $E / N$ of $120 \mathrm{Td}$.

b) LoD determined for $20 \mathrm{~s}$ data collection 


\section{Figure captions}

1. Relative proportions (\%) of $\mathrm{H}_{3} \mathrm{O}^{+}$and $\mathrm{H}_{3} \mathrm{O}^{+}\left(\mathrm{H}_{2} \mathrm{O}\right)$ as a function of $E / N$ when the drift tube was operated in the DC-only mode.

2. Relative proportions (\%) of $\mathrm{H}_{3} \mathrm{O}^{+}$and $\mathrm{H}_{3} \mathrm{O}^{+}\left(\mathrm{H}_{2} \mathrm{O}\right)$ as a function of $E / N$ when the drift tube was operated in the RF (funnel-on) mode.

3. Variation of $\mathrm{C}_{2} \mathrm{H}_{5} \mathrm{O}^{+}$(protonated acetaldehyde) and $\mathrm{C}_{2} \mathrm{H}_{3}{ }^{+}$(fragment from protonated acetaldehyde) signals with effective $E / N$ with the ion funnel switched on (RF mode).

4. Normalized ion counts (relative to $10^{6}$ counts for $\mathrm{H}_{3} \mathrm{O}^{+}$) for $\mathrm{MH}^{+}$, where $\mathrm{M}$ is a specific analyte, versus delivered concentration. These data were collected in the RF funnel-on mode at an effective $E / N$ of $150 \mathrm{Td}$. Note that the vertical scale for protonated acetone counts is on the right hand side of the plot, while the scale used for all other compounds is shown on the left. 


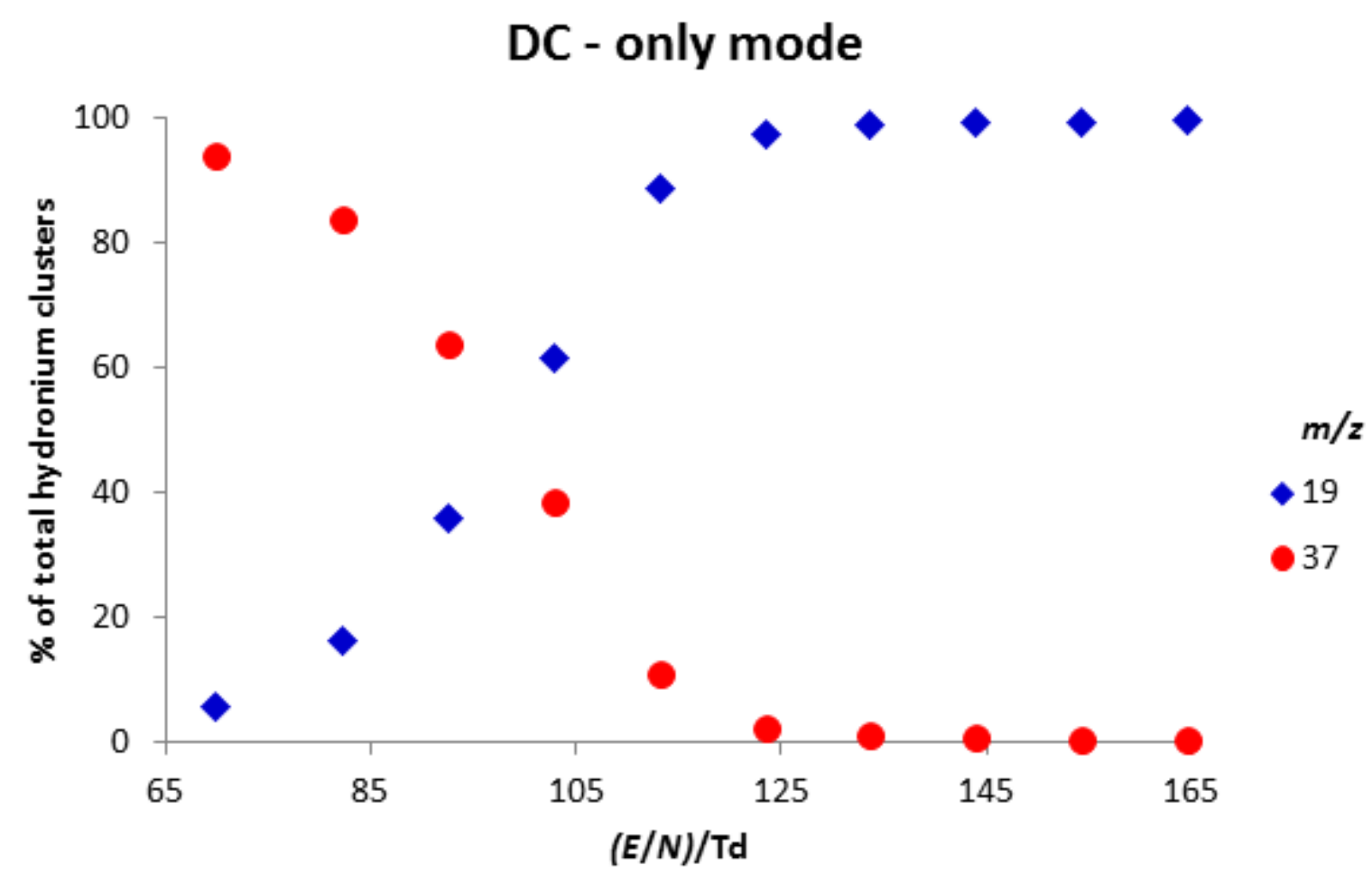

Figure 1 


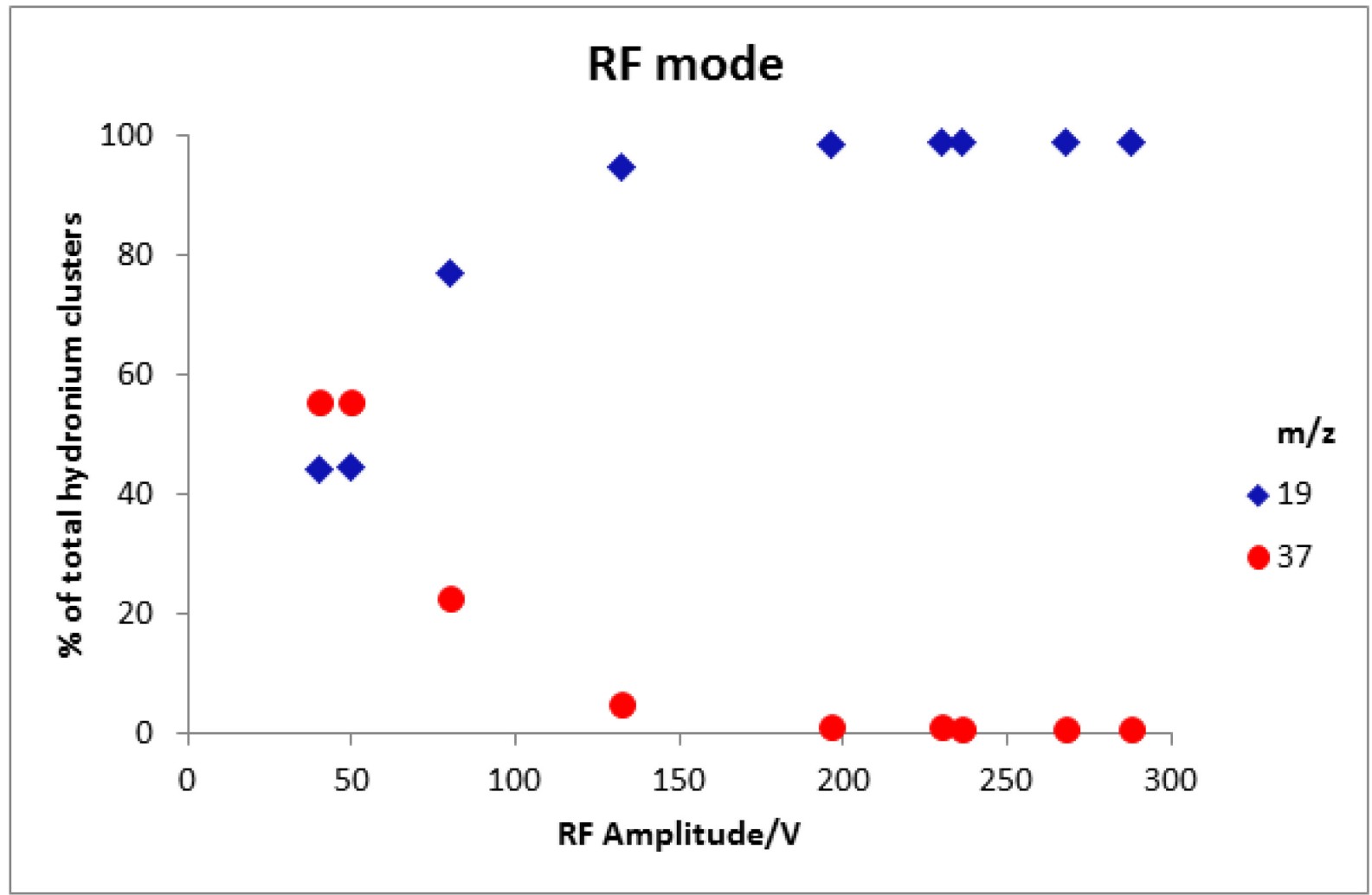

Figure 2 


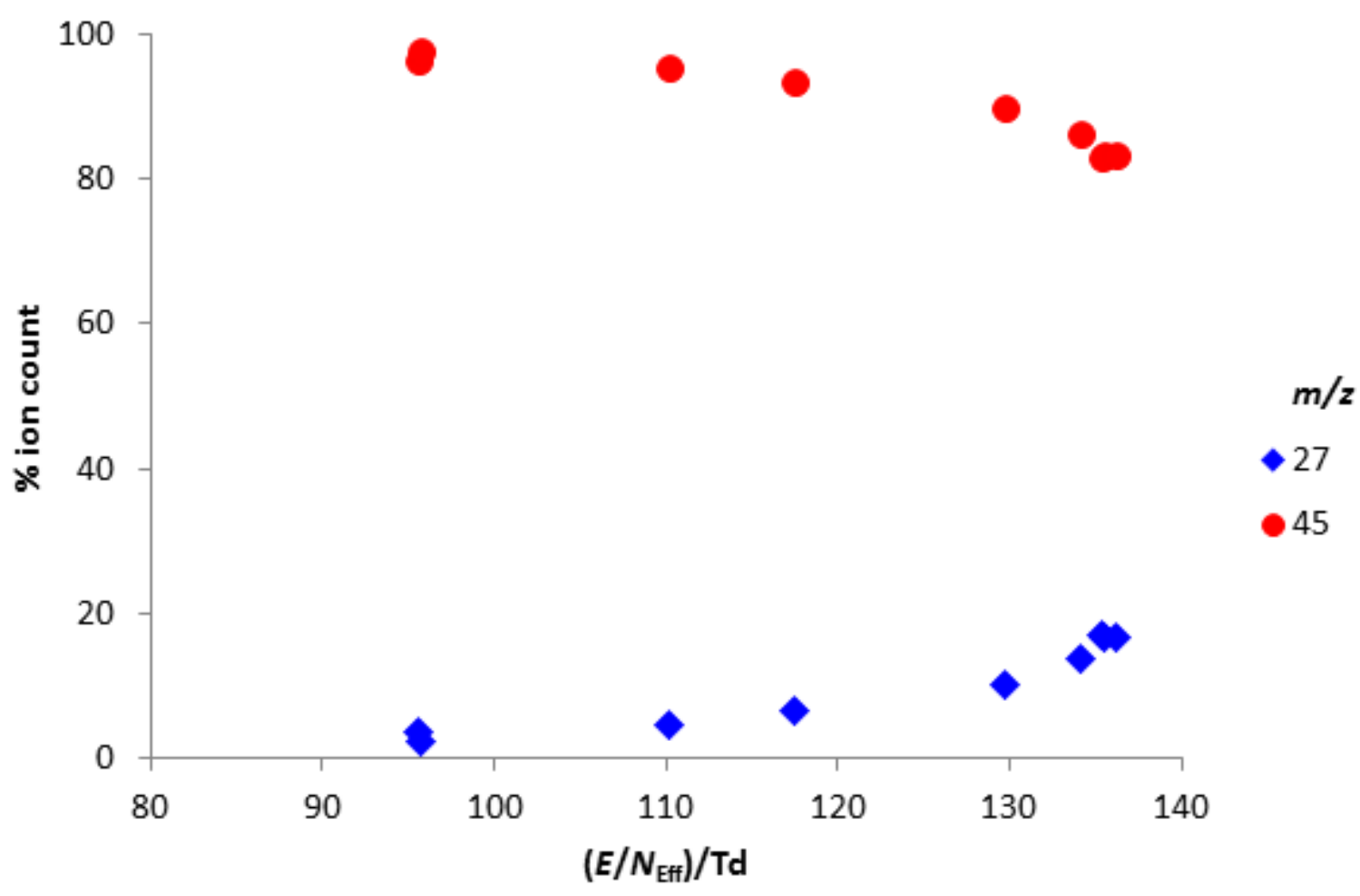

Figure 3 


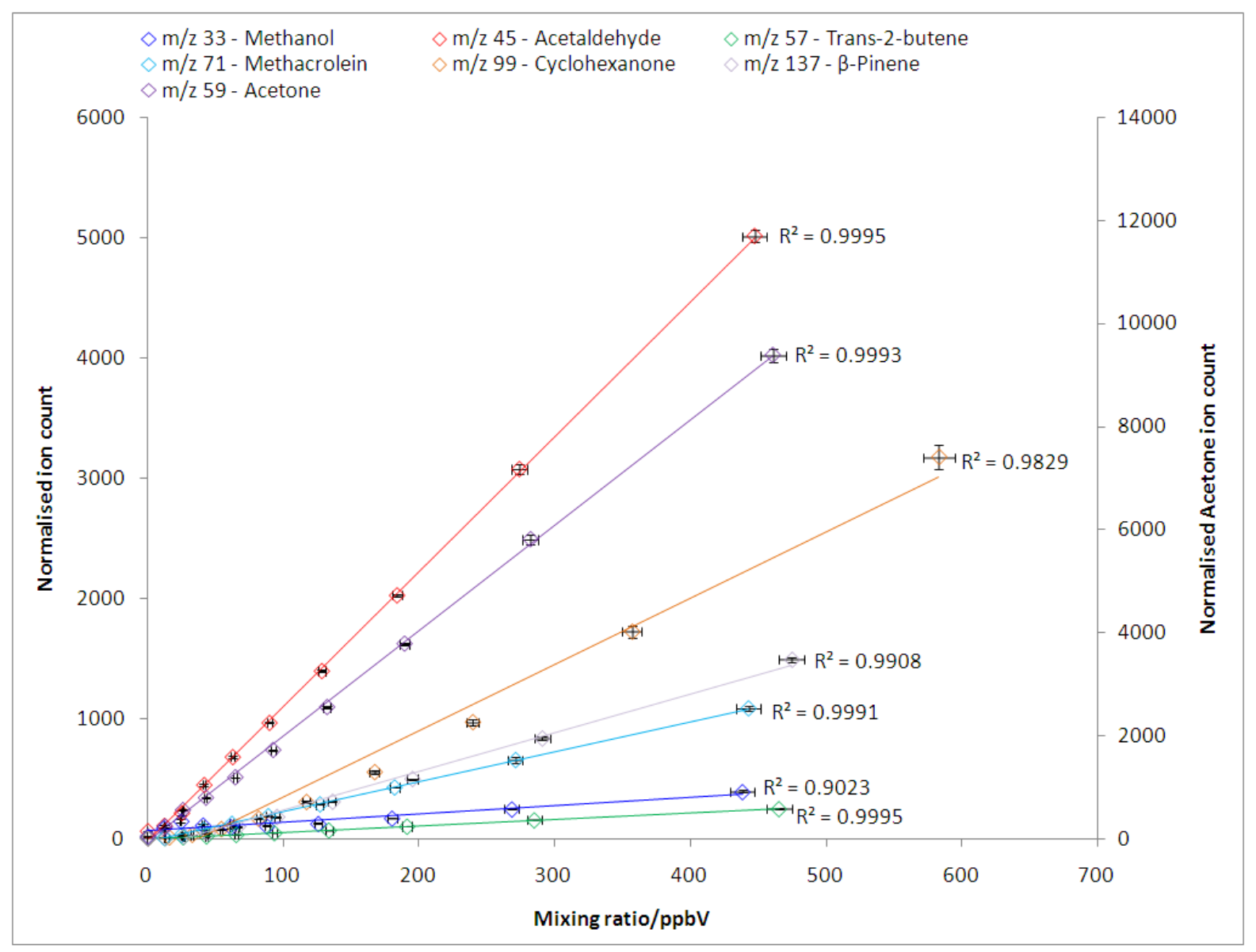

Figure 4 
For TOC only

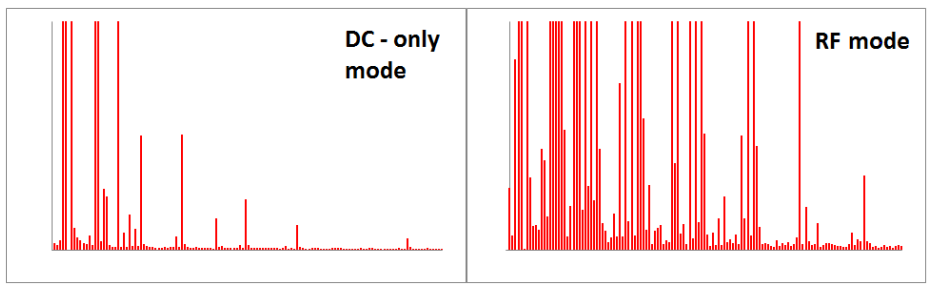

